\title{
A Detuning Study of a Miniaturized PIFA Antenna inside the Human Thorax Model
}

\author{
Amal Bouazizi, Ghada Zaibi, Mounir Samet and Abdennaceur Kachouri \\ Department of Electrical Engineering, LETI laboratory, National School of Engineering of Sfax, University of \\ Sfax, Tunisia
}

\begin{abstract}
Nowadays, the increasing demand for implantable medical devices (IMD) has required the design of efficient invasive antennas. However, designing an antenna operable in a biological layer is actually an extremely challenging task. The human body is not an ideal medium for the transmission of electromagnetic waves. Thus, it degrades significantly the characteristics of implantable antennas coupled with it.

In this paper, the design of a miniaturized slotted rectangular Planar Inverted-F Antenna (PIFA) is proposed. The structure was first optimized to operate in the muscle layer. The location of the antenna was then varied to detect the influence of the other biological layers on its performances.
\end{abstract}

Keywords: biological layers, WBAN, PIFA, interaction

\section{Introduction}

Communication networks, namely wireless networks, have experienced a great evolution for today to focus on, around and inside the human body. With increasingly miniature devices and willing and ambitious users to permanently have access to services that are accessible to them at home and with the aging population and the life expectancy that has considerably increased, a network being part of the fourth generation was developed. This network is known as the Wireless Body Area Network (WBAN). It has attracted worldwide attention because of its great utility.

As a part of a communication system, antennas need to be efficient. However, the problems encountered in antennas applied in medicine, particularly antennas operating inside the human body are different from those of antennas using the free space as a transmission channel. The human body is not an ideal medium for the transmission of radio frequency waves. Consequently, it will be important to highlight the influence of the body tissues on the performance of the antenna. Each biological layer of the human body has its own characteristics in terms of permittivity and conductivity. Biological tissues, usually have high permittivities which change the resonant frequency of antennas coupled with them. The complex environment degrades dramatically the RF antenna performance and a large quantity of the power is then absorbed by the body.

Actually, multiple frequency bands are used for the implanted antennas. Among these bands, we find the Medical Implant Communication Service (MICS 402-405 MHz) [1], the Industrial, Scientific and Medical (ISM 2.4-2.48GHz) [2, 3] as well as the Medical Telemetry System (WMTS 1395-1400 MHz) bands [4]. Indeed, it should be noted that the increase in working frequency which would, to some extent, minimize the dimensions of the antenna will, unfortunately, increase the losses inside the human body. Hence, using the MICS band over the other above cited frequency bands seems rather desirable, given the good propagation characteristics within the human body it exhibits [5] Several antenna solutions for the Wireless Body Area Network are represented in literature, such as the use of the serpentine line or a stacked hook slot [6].The use of spiral antenna [12]. Some of the published solutions focus on the use of PIFA antennas because of their compactness and flexibility in design [7]. This type of antenna is a quarter-wavelength shorted patch which consists of a ground plane, an upper radiator, a coaxial probe and a shorting plate which connects the top radiator to the ground plane. Yet, the detuning effects have already been recognized as a major challenge for the design of implantable antennas [8]-[9]. In [10], the author quantifies the impedance mismatch and the detuning of antennas implanted within different parts of the human body including the head, the trunk, and the limbs. In [11], the author studies the propagation losses of implanted bodily antennas and quantifies the variation in antenna performance when inserted in a single layer and in a multilayer model.

This paper is devoted to the design of a miniaturized PIFA antenna to be implanted within different biological layers. The issue of interactions of the electromagnetic field with biological tissues is presented. This interaction essentially depends on the electrical properties of the surrounding environment.

The remaining of this paper was organized as follows: Section II presents the proposed antenna design. The simulation setup is presented in section III. Results and discussion are highlighted in section IV. Finally, the conclusion is drawn in section $\mathrm{V}$. 


\section{The Configuration of the antenna}

The antenna model geometry is presented in Fig.1. The proposed antenna contains a rectangular radiating element, a ground plane, a substrate and a superstrate. The presented prototype is printed on the Rogers RO 3210 with the specific properties ( $\varepsilon_{r}=10.2$, and thickness $=0.635 \mathrm{~mm}$ ).

To ensure the biocompatibility and to prevent the contact between the antenna and the biological layer, a superstrate layer with the same material of the substrate is used. Rectangular shaped slots are cut on the patch in order to lengthen the current path on the patch surface and consequently miniaturize the antenna. To further miniaturize the dimensions of the antenna, a shorting pin is inserted between the top radiator and the ground plane. The proposed PIFA antenna was simulated using Ansoft High-Frequency Structure Simulator (HFSS) which is a commercial finite element method solver for electromagnetic structures. The antenna parameters are presented in Table 1.

Table 1. The dimensions of the antenna

\begin{tabular}{|c|c|c|}
\hline Parameter & Designation & Value $(\mathrm{mm})$ \\
\hline $\mathrm{h}$ & Height of the substrate & 0.635 \\
\hline $\mathrm{Ls}$ & Length of the substrate & 11.9 \\
\hline $\mathrm{Ws}$ & Width of the substrate & 14.7 \\
\hline $\mathrm{Lg}$ & Length of the ground plane & 11.9 \\
\hline $\mathrm{Wg}$ & Width of the ground plane & 14.7 \\
\hline $\mathrm{Lp}$ & length of the patch & 10.926 \\
\hline $\mathrm{Wp}$ & Width of the patch & 14.36 \\
\hline $\mathrm{Lsl}$ & length of the slot & 9.81 \\
\hline $\mathrm{Wsl}$ & Width of the slot & 0.5 \\
\hline $\mathrm{Rp}$ & Radius of the pin & 0.3 \\
\hline $\mathrm{t}$ & Height of the conductor & 0.035 \\
\hline
\end{tabular}

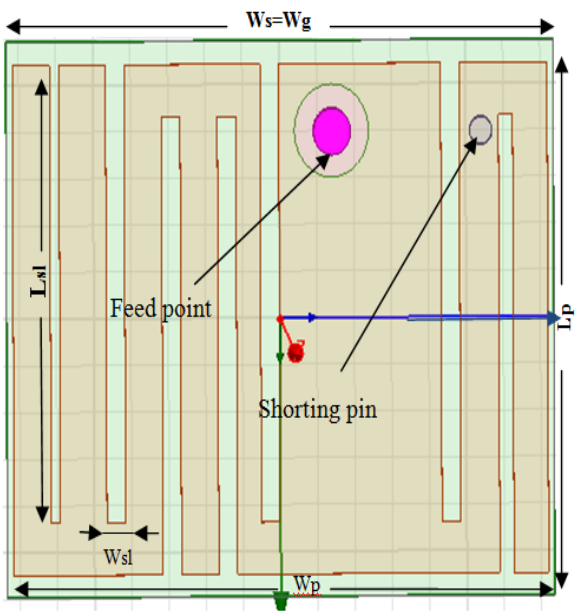

Fig. 1. The configuration of the proposed antenna

\section{Simulation setup}

Designing an antenna inside the human body is really a difficult task. For a practical antenna design, many factors have to be taken into account like the biological layers properties and their shapes.

The thorax model, including the muscle, the skin, and the fat layers is used to study the interaction between the electromagnetic field and the body tissues. Each of these tissues has its own characteristics in terms of permittivity and conductivity as shown in Table2.

Table. 2 The dielectric properties of Skin, fat, and muscle [13]

\begin{tabular}{|c|c|c|}
\hline Tissue & Conductivity (S/m) & Permittivity \\
\hline Skin & 0.68934 & 46.718 \\
\hline Fat & 0.041167 & 5.5785 \\
\hline Muscle & 0.79708 & 57.104 \\
\hline
\end{tabular}

The structure has been first optimized to work in the muscle layer. The location of the antenna was then varied to detect the influence of the other layers on its functioning as shown in Fig.2
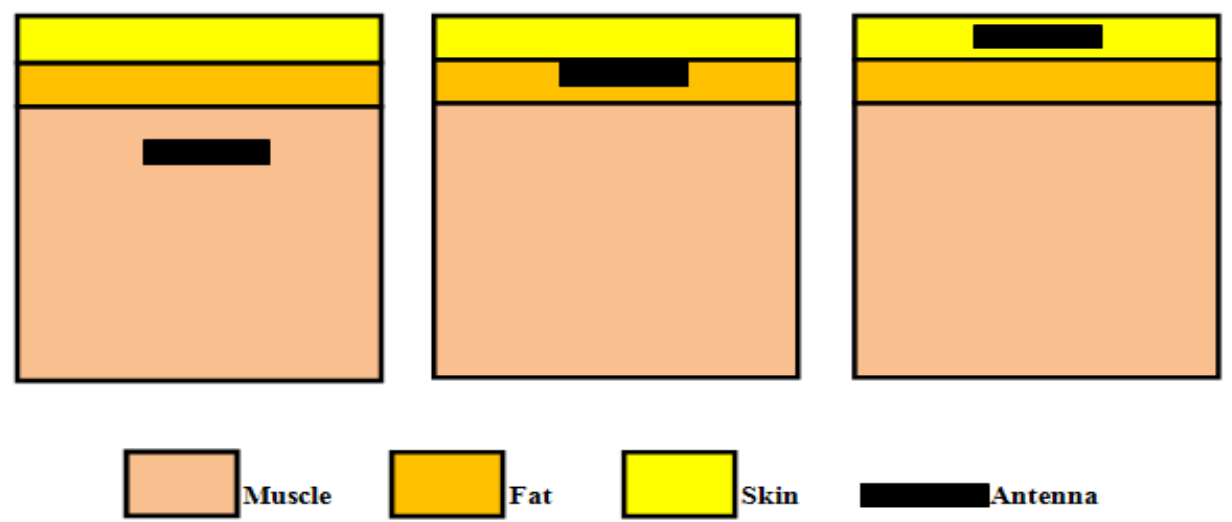

Fig. 2. The position of the antenna within the physical model 


\section{Results and discussion}

This section will be focused to determine the characteristics of the simulated antenna in terms of coefficient reflection (S11), Bandwidth, Z parameter as well as the radiation pattern when it is implanted within different biological layers.

\section{A. Reflection coefficient}

The reflection coefficient is a fundamental parameter which is equal to the ratio of the reflected wave amplitude to the incident wave. Fig.3 shows the variation of the simulated reflection coefficient of the antenna as a function of frequency. As appeared in the figure, the resonance frequency is not fixed. The antenna has a frequency of $403 \mathrm{MHz}$ when it is positioned within the muscle layer and its return loss is equal to $-40.52 \mathrm{~dB}$. Then a frequency shift towards higher bands is noticed when the antenna was placed in the skin layer. The obtained frequency is $421 \mathrm{MHz}$ with a reflection coefficient equal to $-17.96 \mathrm{~dB}$. Finally, a frequency of 505 $\mathrm{MHz}$ is achieved with a reflection coefficient of $-1 \mathrm{~dB}$ when the antenna was positioned within the fat layer.

The obtained results from inside the thorax change significantly according to the antenna positions. The resonant frequency of the antenna depends on the permittivity of the biological layer. The fat layer with the smallest value in terms of permittivity compared to the skin and muscle layers presents the highest frequency shift towards higher frequencies.

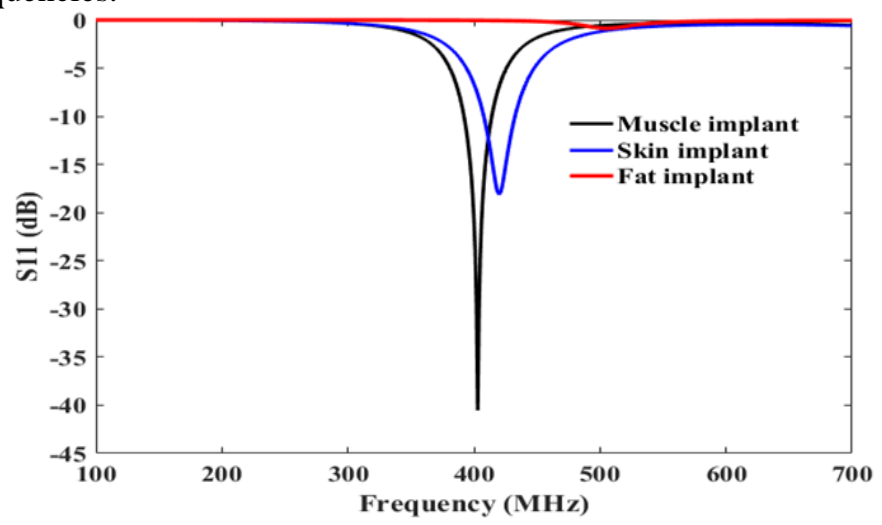

Fig. 3.The reflection coefficient versus frequency of the antenna within the muscle, the skin and the fat

\section{B. Bandwidth}

The term bandwidth specifies the range of frequencies that an antenna can reach in order to obtain a desirable behavior with certain characteristics. The bandwidth can be considered as the frequency range on either side of a central frequency.

$$
B W=f_{2}-f_{1}
$$

Today, the miniaturization of medical electronic devices requires the use of miniaturized antennas. As a result, they are expected to have a narrow bandwidth. Unlike antennas in the free space, implantable antennas can have wide bandwidths due to the losses introduced by the surrounding tissues. In this section, we try to study the influence of the different layers on the antenna's associated bandwidth which is calculated at $-10 \mathrm{~dB}$. Fig. 4 shows the obtained bandwidth of the antenna within the different layers. As appeared in the figure, when the antenna was placed within the skin layer its bandwidth has a value of $24 \mathrm{MHz}$ compared to a value of 22 $\mathrm{MHz}$ in the muscle layer. This can be explained by the increase of losses within the skin. The calculated bandwidth within the fat layer cannot be mentioned because the antenna reflection coefficient is only $-1 \mathrm{~dB}$.

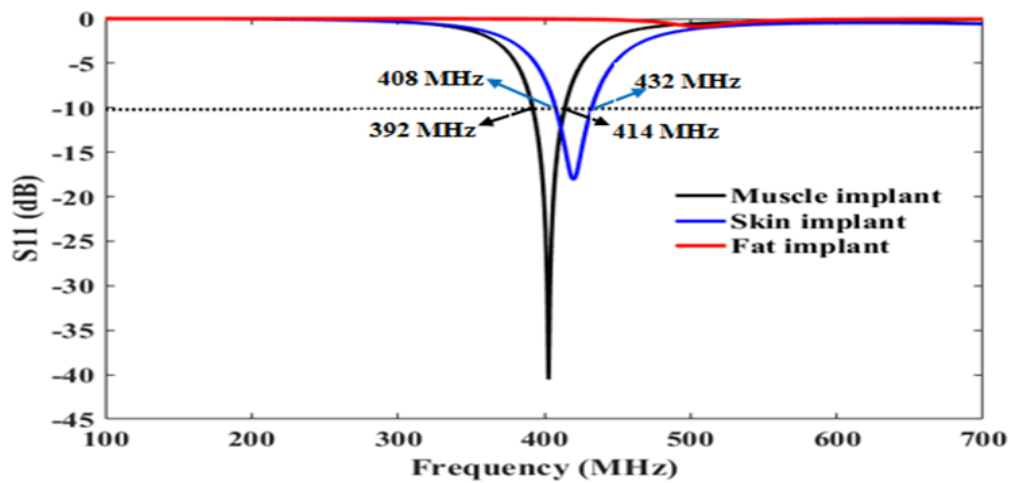

Fig. 4. The bandwidths of the antenna within the muscle, the skin and the fat 


\section{Z parameter}

The impedance ( $\mathrm{Z}$ parameter) is the ratio between the voltage and the current at the input of an antenna. The usefulness of this concept is important to ensure the best energy transfers between the antennas and the devices connected to them.

The impedance of an antenna can be given by the following equation:

$$
Z=R+j X
$$

Where $\mathrm{R}$ and $\mathrm{X}$ are the resistance and the reactance of the antenna respectively. Fig. 5 and Fig.6 show the variation of the antenna resistance and reactance, respectively, according to the specific layer.

The impedance value is around the ideal value $(50+\mathrm{j} 0) \mathrm{Ohm}$ when the antenna was placed within the muscle layer. However, the antenna impedance is dramatically affected by the fat layer.

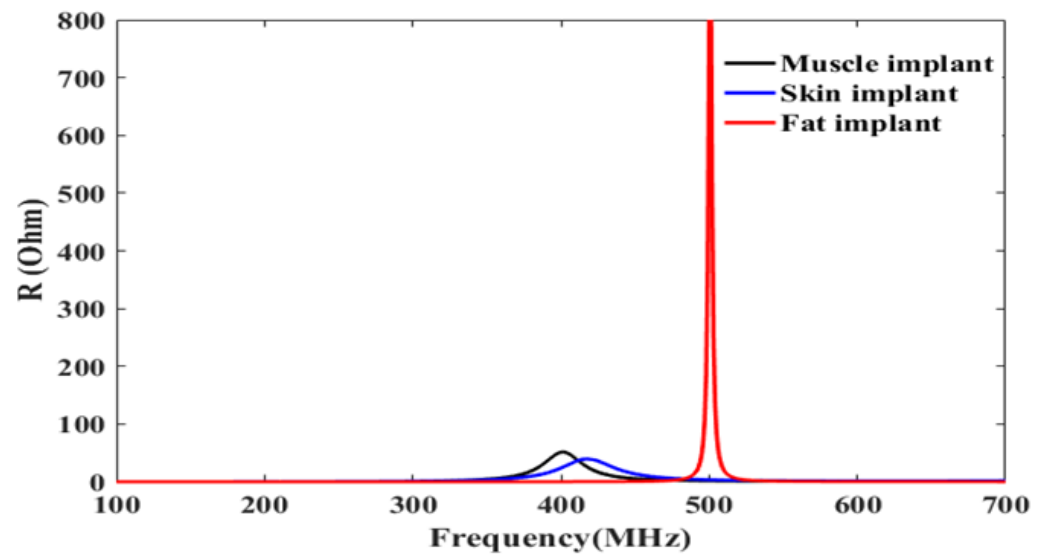

Fig.5. The antenna's resistance within the muscle, the skin and the fat

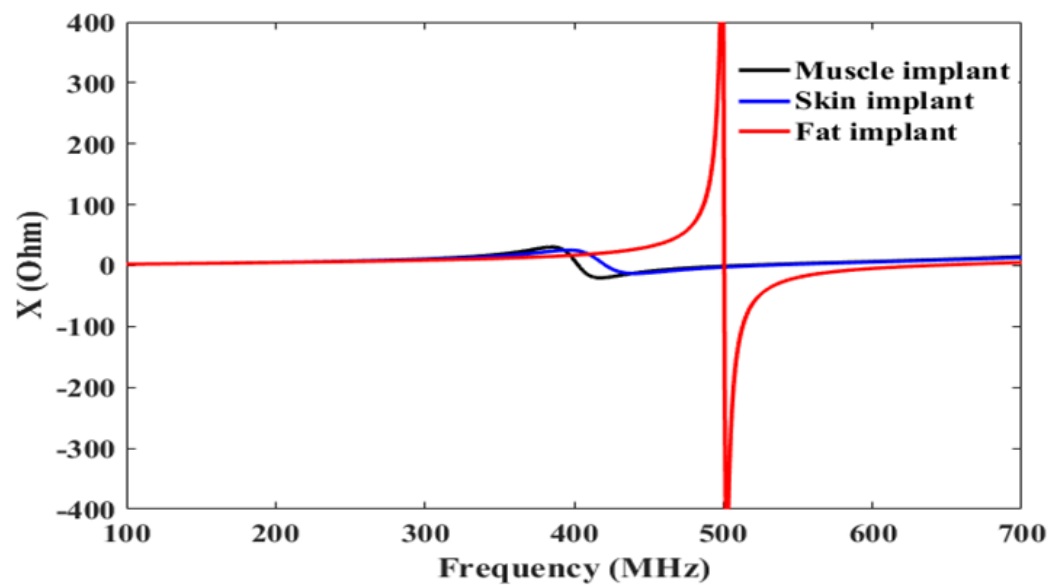

Fig. 6. The antenna's reactance within the muscle, the skin and the fat

Overall numerical results of the $\mathrm{Z}$ parameters (resistance and reactance) extracted from Fig. 5 and Fig. 6 for the different positions appear in Table 3 .

Table 3. The antenna's $\mathrm{Z}$ parameters within the different layers

\begin{tabular}{|c|c|c|}
\hline Tissue & $\mathrm{R}(\mathrm{Ohm})$ & $\mathrm{X}(\mathrm{Ohm})$ \\
\hline Skin & 38.8 & 0.78 \\
\hline Fat & 84.5 & -247 \\
\hline Muscle & 50.9 & -0.09 \\
\hline
\end{tabular}

\section{Radiation pattern}

The radiation pattern is a graphical representation. It describes the spatial distribution of the power radiated by the antenna. 
Fig. 7 (a) and Fig.7 (b) show the simulated radiation pattern of the antenna for both planes $\varphi=0^{\circ}$ and $\varphi=90^{\circ}$, respectively. Based on the reached results, one could well note that the radiation pattern depends on the specific position of implantation. For both planes $\varphi=0$ and $\varphi=90$, the antenna has an omnidirectional radiation pattern when it is placed within the muscle layer. However, it has a directional radiation pattern when it is placed within the skin and the fat layer

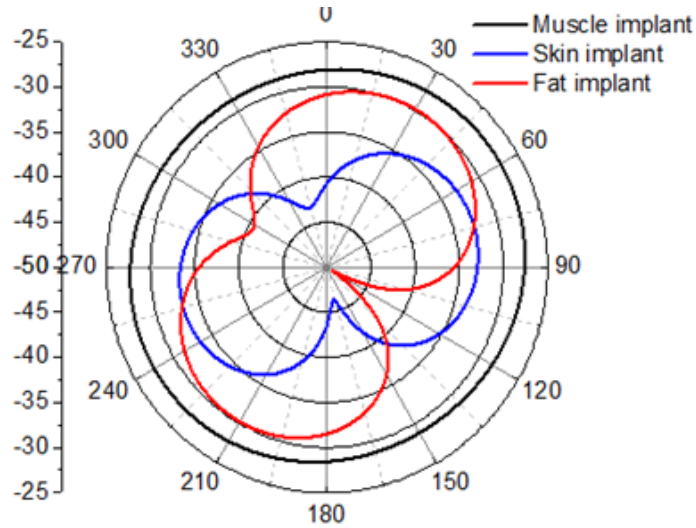

(a)

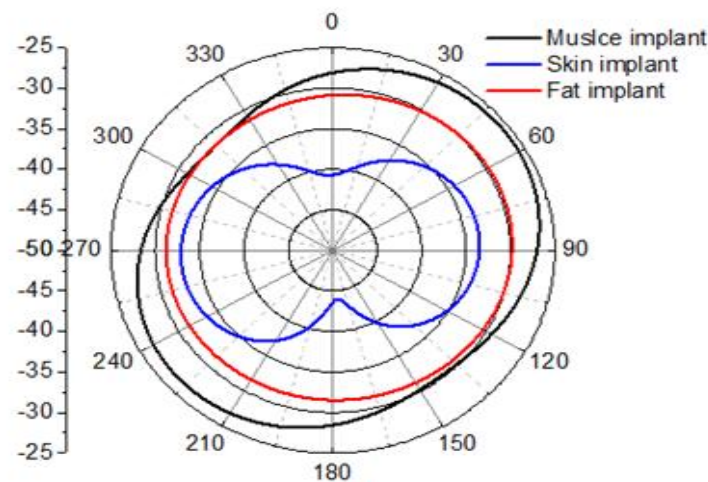

(b)

Fig. 7. The radiation pattern of the antenna within skin, fat and muscle layers : (a) $\varphi=0$, (b) $\varphi=90$

\section{Conclusion}

In this paper, we have presented a PIFA antenna design. The proposed structure was positioned within different layers including the skin, the fat and the muscle layers. When placed within the muscle layer, the antenna resonated at $403 \mathrm{MHz}$. However, the positioning of the structure within the fat and the skin layers produced a frequency shift towards higher bands due to the low permittivity of these layers compared to the muscle. The bandwidth, the $\mathrm{Z}$ parameters as well as the radiation pattern depend on the surrounding medium. All these results confirm that the use of an implantable antenna for a specific application depends on the implantation medium.

\section{References}

[1] A Kiourti, M. Christopoulou, and K.S Nikita, Performance of a Novel Miniature Antenna Implanted in the Human Head for Wireless Biotelemetry, IEEE International Symposium on Antennas and Propagation, Spokane, Washington, July 2011, 392-395

[2] J. Kim, and Y. Rahmat-Samii, Implanted antennas inside a human body: Simulations, designs, and characterizations, IEEE Trans. Microw. Theory Tech, 52 (8), 2004, 1934-1943.

[3] W. Xia, K. Saito, M. Takahashi, and K. Ito, Performances of an implanted cavity slot antenna embedded in the human arm, IEEE Trans. Antennas Propagation, 57(4), 2004, 894-899.

[4] J. Ung, and T. Karacolak, A Dual-Band Meandered Dipole Antenna for Medical Telemetry Applications Progress In Electromagnetics Research C, 63, 2016, 85-94.

[5] FCC Rules and Regulations. MICS Band Plan. Part 9, Jan.2003.

[6] W.C.Liu, S.H.Chen, and C.M.Wu, Implantable Broadband Circular Stacked PIFA Antenna for Biotelemetry Communication, Journal of Electromagnetic Waves and Applications, 22 (13), 2008, 1791-1800.

[7] O.K Hammouda. and A. M. M.Allam, Utilizing Implanted Antennas to Detect the Presence of Oral Cancers, Research Journal of Engineering Sciences, 3 (7), 2014, 22-27. 
[8] C.M Furse, Biomedical telemetry: Today's opportunities and challenges. IEEE Workshop on Antenna Technology Small Antennas and Novel Metamaterials, Santa Monica, California, USA, Mar. 2009,1-4.

[9] P. Soontornpipit, C. M.Furse, and Y. C Chung, Design of implantable microstrip antennas for communication with medical implants; IEEE Transactions on Microwave Theory and Techniques, 52 (8), 2004, 1944-1951.

[10] N.Vidal, S.Curto, J M .Lopez Villegas, J.Sieiro, and F. M Ramos, Detunning Study Of Implantable Antennas Inside The Human Body, Progress In Electromagnetics Research, 124, 2012, 265-283.

[11] J. Gemio, J. Parron, and J. Soler, Human Body Effects on implantable antennas for ISM bands applications: Models Comparison and propagation losses study, Progress In Electromagnetics Research, 110, 2010, 437-452.

[12] Jae-Ho Lee, Dong-Wook Seo, and Hyung Soo Lee, Design of Implantable Rectangular Spiral Antenna for Wireless Biotelemetry in MICS Band, ETRI Journal, 37 (2), 2015, 204-211.

[13] http://niremf.ifac.cnr.it/tissprop/htmlclie/htmlclie.php. 